\title{
Importance of the diagnosis of bipolar mixed states
} Olavo Pinto

\author{
Address: Clinica de Neuropsiquiatria, Rio de Janeiro, Brazil \\ from International Society on Brain and Behaviour: 3rd International Congress on Brain and Behaviour \\ Thessaloniki, Greece. 28 November - 2 December 2007 \\ Published: 17 April 2008 \\ Annals of General Psychiatry 2008, 7(Suppl I):S90 doi:I0.II86/I744-859X-7-SI-S90
}

This abstract is available from: http://www.annals-general-psychiatry.com/content/7/SI/S90

(c) 2008 Pinto; licensee BioMed Central Ltd.

Mixed states are an affective condition in which manic and depressive symptoms are present at the same time. In the original description by Kraepelin and Weygandt features of mania and depression did coexist in the psychopathological domains of mood, cognition and psychomotor activity. Due to the great influence of American psychiatric nosology, the DSM system, a lot of interest has been placed on manic variations of mixed states under dysphoric mania, with intrusion of full blown depressive symptoms into mania. We will focus on a neglect aspect of mixed states, its presence in depression, depressive mixed state, and its enormous importance in the clinical setting. Bipolar depressive mixed states are more chronic, more frequent, more serious, highly comorbid with psychiatric and medical conditions and generate increased suicidality. The presence of hypomanic features in depressed bipolar II patients is fairly common in any given psychiatric outpatient population. This could generate a very complex and ever changing clinical picture of affective instability, fluctuation, liability, irritability and diurnal variation that are mostly sustained over time. It is the great imitator of our era, like tuberculosis was for internal medicine during the first half of the last century. Differential diagnosis could be made with multiple psychiatric condition like unipolar agitated depression, schizophrenia, borderline personality disorder and also clinical states like delirium. The implications of mixed states recognition are of utmost clinical practice importance. The widespread use of massive and multiple antidepressant pharmacotherapy, do generate/worsen mixed states. It would be central to distinguish mixed states from other conditions avoiding treatments that do worsen symptomatology, e.g. antidepressants, and using others that might be effective, e.g. anticonvulsants, atypical antipsychotics and eletroconvulsive therapy. 